\title{
THE SETTINGS APPROACH TO ACHIEVING BETTER HEALTH FOR CHILDREN
}

\author{
Lesley King \\ Centre for Public Health, University of NSW, Sydney
}

This article describes the rationale supporting the use of a settings approach to improve children's health. School is one key setting for promoting health, and so the concept of health promoting schools has been developed by the World Health Organization. The article refers to examples of how the model has been adapted for Australia.

A settings approach is a neat way of packaging interventions and actions to improve children's health, for both practical and theoretical reasons. A settings approach locates public health action in the social, cultural and physical places in which children live, learn and play. This approach has been popularised and applied to health promoting schools, healthy cities, healthy localities, and most recently 'healthy islands'.

A setting refers to a socially and culturally defined geographic and physical area of social interaction, and a socially and culturally defined set of patterns of interactions performed in the area. ${ }^{2}$

Theoretically, the settings approach has much to offer, for it adopts a social-ecological perspective, which recognises that health is influenced by contextual and environmental factors. What logically follows is an approach to problem solving and action that involves addressing the range of physical, social, organisational and cultural factors influencing health in an environment. Settings are therefore more than convenient locations for reaching target groups; they are also social systems that can support health and provide avenues for changing social systems, not just individuals. The types of outcomes expected from working in this way include changes in environments, policy, skills and organisational processes, as well as changes related to specific health problems.

From a practical point of view, the settings approach does not preclude a focus on specific health issues, and can serve two purposes:

- addressing a specific health problem

- developing the general problem solving capacity of the organisations involved in that setting. ${ }^{3,4}$

The settings approach offers an alternative to vertical ways of structuring programs, in which separate programs address individual health problems (such as heart disease and injury). Such an integrated approach ensures greater coordination in negotiating with stakeholders and in the compilation of resource materials. Reduced duplication of effort and competition between programs are other potential benefits. ${ }^{5}$

\section{HEALTH PROMOTING SCHOOLS}

The school has traditionally been an attractive setting for health promotion, for it provides a way of reaching a large proportion of children and young people, and brings with it a team of professional educators. In recognising the school as a social system, the opportunity arises to influence structural aspects of the school environment and adopt a more comprehensive approach to improving health. The health promoting school concept has been promulgated by the World Health Organization, and programs have been implemented in many countries, including Canada, the United Kingdom, the United States and Australia.

Health promoting schools have been characterised as having six domains for action: the formal curriculum, the school ethos, school policies and practices, school health services, school-home-community interactions, and organisational structures. ${ }^{6}$ The evidence regarding interventions to improve children's health indicates the value of intersectoral, comprehensive programs, and these can be developed and implemented through the structure of health promoting schools programs. ${ }^{7}$

While the concept of health promoting schools is well developed, the practice lags. We are currently grappling with how to achieve widespread implementation of a health promoting schools program, and how systematically to monitor progress and results. A key feature of the program as it has been implemented in Australia is the collaboration between the health and education sectors, which has occurred (to varying degrees) at national, State and local levels. The Western Australian School Health program (WASH), an intersectoral program providing a model of operation, professional development and followup support to schools, is a well-documented example of a State implementation program. ${ }^{8,9}$ In NSW, as part of the Coalfields Healthy Heartbeat program, a health promoting schools project was conducted with 15 primary schools in a socially disadvantaged region. ${ }^{10}$ Consistent with 
findings from studies on intersectoral collaboration generally, a key lesson from local health promoting schools programs has been the importance of tailoring activities to complement core school activities. ${ }^{10}$

Implementation of health promoting schools programs requires commitment from both education and health sectors and an orchestrated approach. As a new, evidencebased policy and program initiative, the health promoting schools approach requires infrastructure and resources-such as workforce development and technical support - if it is to be widely implemented.

A further essential ingredient for successful implementation is enthusiasm and initiative at the local level. The concepts of health promoting schools, and settings generally, have been found to be very motivating for professional and lay groups. They offer a positive and participatory approach to health, engaging all stakeholders in the task of making better environments and organisations. The challenge now is to harness this interest, to develop a strong implementation and actionresearch program, to monitor (and adjust) ways of building organisational capacity, and to study how this translates over time into improved health.

\section{REFERENCES}

1. Ritchie J, Rotem A, Hine B. Healthy Islands: from concept to practice. Pacific Dialogue, 1998. In press.

2. Wenzel E. A comment on settings in health promotion. Internet J Health Promot http://www.monash.edu.au/health/ IJHP/1997/1. Accessed 7 May 1998.

3. Grossmann R, Scala K. Health promotion and organizational development: developing settings for health. Vienna: WHO Regional Office for Europe, 1993.
4. Hawe P, Noort M, King L, Jordens C. Multiplying health gains: the critical role of capacity-building within health promotion programs. Health Policy 1997; 39: 29-42.

5. Tannahill A. From priorities to programmes. Health promotion country series, no. 1. Edinburgh: Health Education Board for Scotland, 1994.

6. Booth ML, Samdal O. Health-promoting schools in Australia: models and measurement. Aust N Z J Public Health 1997; 21(4): 365-370.

7. Alperstein G, Thomson J, Crawford J. Health gain for children and youth of Central Sydney. Strategic plan. Sydney: Health Services Planning Unit and Division of Population Health, Central Sydney Area Health Service, 1996.

8. Cameron I, McBride N. Creating health promoting schools: lessons from the Western Australian school health project. Health Promot J Aust 1995; 5(1): 4-10.

9. McBride N, Cameron I, Midford R, James R. Facilitating health promotion in Western Australian schools: key factors for success. Health Promot J Aust 1995; 5(1): 11-16.

10. Williams P, Weston R, McWhirter J, et al. Health promoting schools: lessons from working intersectorally with primary schools in Australia. Health Educ J 1996; 55: 300-310.

\section{Correction}

An incorrect phone number was published in the October 1998 issue of the Bulletin. The correct phone number for the Population Health Unit on the Mid North Coast is (02) 65830750 . The editor apologises for any inconvenience caused. 